\title{
IoT Enabled Low Cost Wearable Device for Location and Information Tracking System
}

\author{
Nur Atika Binti Kamaludin ${ }^{1 *}$,Normaliza Omar ${ }^{l}$, Thennarasan Sabapathy ${ }^{l}$, Nursabrina Binti Iskandar ${ }^{1}$, and Muhammad \\ Ramlee Kamarudin ${ }^{2}$ \\ ${ }^{1}$ Biolectromagnetic Research Group, School of Computer and Communication Engineering, Universiti Malaysia Perlis (UniMAP), \\ Malaysia \\ ${ }^{2}$ Centre for Electronic Warfare, Information and Cyber (EWIC), Cranfield Defence and Security, Cranfield University.
}

\begin{abstract}
An internet-of-things (IoT) enabled low cost wearable device for location and information tracking system is developed to monitor and ensure the safety of the children while their parents are away either to work or abroad. This system aims to ensure human safety especially the children and efficiently initiate search and rescue in the event of emergencies. The device will notify the parents on the condition of the surroundings of the children, specifically inside the house and notify the parents if the children are outside the target area by using the application developed in the smartphone. The system uses a microcomputer called Beagle-Bone Black (BBB) as the processor while GPS and LTE technology for wireless communication. Future improvement mainly focuses on reducing the size and power consumption of the device, improving communication module, and optimizing microcomputer's specification.
\end{abstract}

\section{Introduction}

Generally, tracking system is the system that is used to track any object or human at any location. Nowadays, this system is very popular to track anything at any time. Recently, there many techniques and technologies can be using to develop and improve this system. The aim of this work is to ensure the safety of the children while their parents are away either to work or abroad. The proposed system will notify the parents on the condition of the surroundings of the children which specifically targeted inside the house and notify the parents if the children are outside the target area.

Nowadays, there are many techniques and technologies can be implanted in tracking system. Various platform has been used for such location tracking system for home users [1]. However, these systems are expensive and has high complexity. Some of the system are also does not support internet-of-things (IoT). The proposed location tracking system in this work uses latest technologies to make it user friendly. It supports IoT and adopts geo-fencing [2] techniques to alert the parents about the location of the children. The development of the proposed system is further explained in following section.

\section{Methodology}

Methodology discusses about the overall components involved in the proposed system. Mainly these components divided to five parts such as M-WiCom, As shown in Figure 1, the component used it is Beagle-Bone
Black also known as M-WiCom for the operation system and smartphone for parents to monitor the position of their children. Physically from that figure, Beagle-Bone Black (BBB) divided into three parts of antenna. There are GPS, LTE and RF module [3]. But in this work, GPS and LTE are only being used. For the GPS antenna will connect with GPS satellite. The function of GPS is to monitor the coordinate or the position of their children. For the LTE antenna is to transmit the information about the children through the smartphone. In this BeagleBone Black, there is processor that that control all the system in the Beagle-Bone Black.

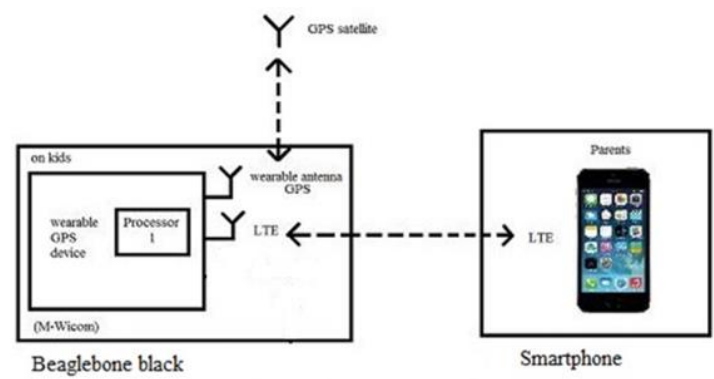

Fig. 1. System concept.

The flowchart in Figure 2 shows the operational flow of the whole system. It starts with setting the current GPS coordinate as origin. Then it specifies radius limit to 100 meters. Next get the latest GPS coordinate will be obtained when the user moves. Whenever the children move, it can read the distance for the movement of the children. The notification will be sent when the distance current GPS coordinate minus the set current GPS

* Corresponding author: atikakamaludin94@gmail.com 
coordinate as origin more than 100 meters. When the notification on, it will directly send information to the parents through the LTE by using smartphone.

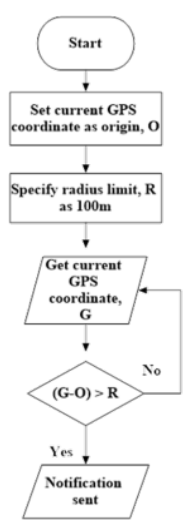

Fig. 2. Flowchart of the system operation.

\subsection{Location tracking system using M-WiCom (GPS and LTE enabled)}

The Figure 3 shows the M-WiCom. This location tracking system uses $\mathrm{M}-\mathrm{WiCom}$ which is based on Beagle-Bone Black (BBB) as the processing platform. M-WiCom is selected because it GPS and support with LTE on a single board. Thus it could reduce the complexity of the design where the use of many devices can be avoided.

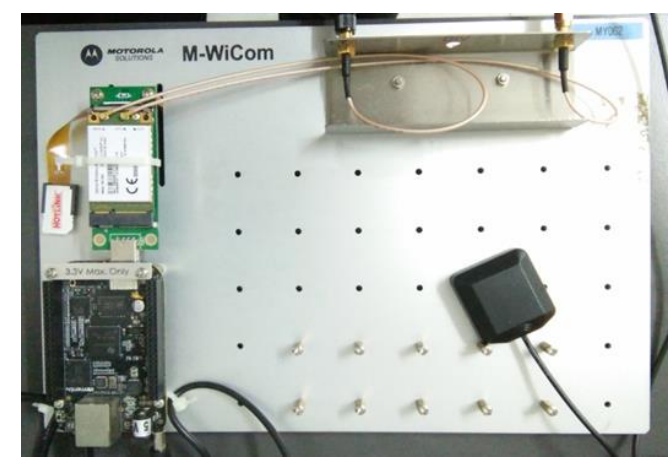

Fig. 3. M-WiCom.

\subsubsection{Data Processor}

Figure 4 shows the Beagle-bone Black Microcomputer. A microcomputer called Beagle-Bone Black (BBB) with $1 \mathrm{GHz}$ processing speed and $512 \mathrm{MB}$. The microcomputer can perform similarly to a regular computer. It can boot with various operating systems which are easily available. This microcomputer is used for the following reasons:

1. The board has UART serial interface which is suitable with RF transceiver.

2. It has a USB port which enables it to be connected to a 4G LTE module.
3. The board can perform network programming like regular computer, thus enables it to access external databases.

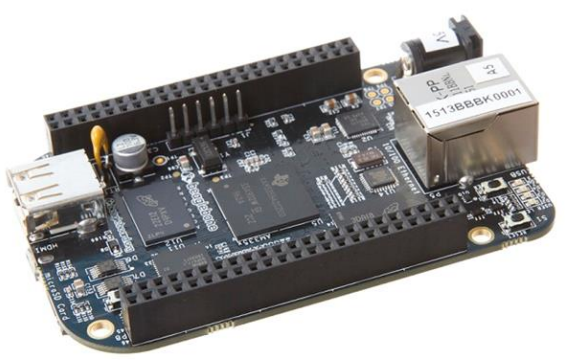

Fig. 4. Beagle-Bone Black Microcomputer.

On the other hand, modem is needed for the data processor to connect to the internet. For the purpose of providing mobile internet connection, an embedded wireless module is used. The wireless module is Sierra MC7304 as shown in Figure 5, support both 3G/4G LTE and GPS communication. This module is attached to a USB adapter with a SIM card slot which is then connected to the data processor via its USB port.
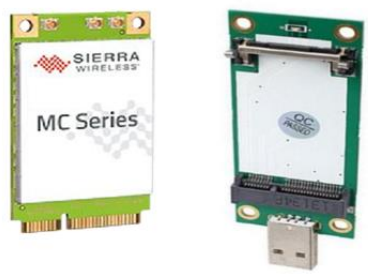

Fig. 5. Wireless internet with mini PCI-E to USB adapter.

Figure 6 shows the SIM card slot. The SIM card stores data such as user identity, location and phone number, network authorization data, personal security keys, contact lists and stored text messages [2].

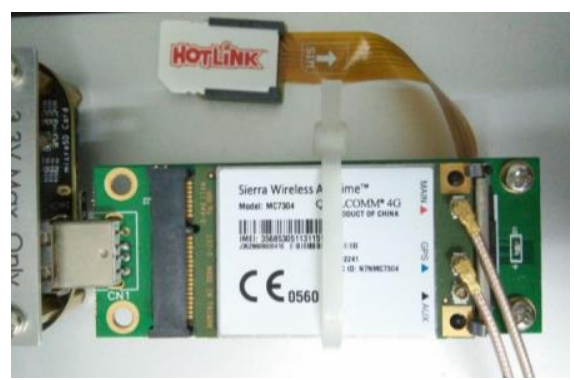

Fig. 6. SIM card slot.

The operating system used in BBB is Ubuntu Linux. Figure 7 shows the block diagram that illustrating the role of microcomputer. The GPS coordinates received by the BBB. Then according to the processed data, the LTE (4G) communication will be established if the notification needed to be sent to the parent's mobile. 


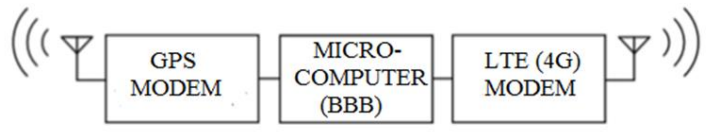

Fig. 7. Microcomputer architecture block diagram.

\subsubsection{GPS communication device and LTE modem}

In this work, the communication device of GPS and LTE are proposed to use. Communication device of GPS is used to monitor the position of the children. Meanwhile, the communication device of LTE is used to send information to the parents. Both techniques are suitable to make some improvement and development of the tracking system.

The GPS is used for tracking the location. There are three ways to target location. Figure 8 shows the ways to target location. The geo-fencing method is selected to give a certain coverage for the user. Geo-fencing is a virtual perimeter and virtual barrier for geographical areas [4]. It could be dynamically generated as in a radius around a store or point location. In this proposed framework, $100 \mathrm{~m}$ radius is selected around the center point of a location. Notification will be send to parents when the children move out from the radius value.
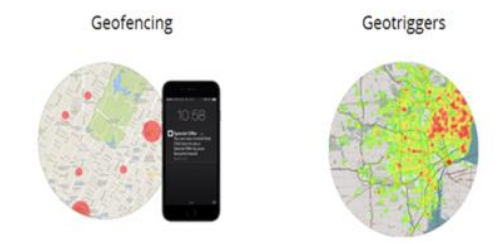

Fig. 8. Ways to target location.

Long Term Evolution or known as LTE is one of the communication devices for this tracking system. This Mobile terminal modem unit interfaces with cellular base stations and send or receives user information data generated by the application unit. So, it interacts with a base station using different cellular interface standard like GSM, LTE or others to send or receive information to distantly located, called server. It also interacts locally with its applications units, like data transfer applications for getting or providing the user application data [5]. LTE is used to send information from M-WiCom to the smartphone.

\subsection{Smartphone (Notification to parents)}

The use of the smartphone in this system is to receive the notification from the M-WiCom. MIT apps inventor will be developed for the smartphone to receive the notification. MIT Apps Inventor is an innovation beginner's introduction to programming and app creation that transforms the complex language of text-based coding into visual, drag and drop building blocks. The simple graphical interface grants even an inexperienced novice the ability to create a basic, fully functional app within an hour or less. The android apps will be developed using Android. Any information about the position their children can be known through this smartphone. Through that application, the parents can monitor their children's location. Figure 10 shows the application in the smartphone.

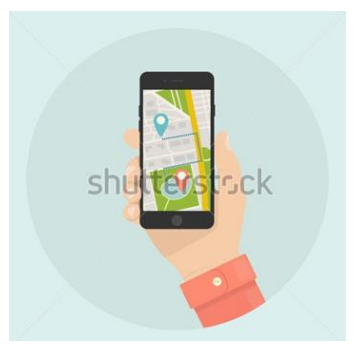

Fig.10. Application in the smartphone.

\subsection{Software Development}

In this section, the stage by stage development of the software part is explained. It start with the software development in BBB by using Linux to Apps development for mobile phone. A detailed explanation is given in the following subsections.

\subsubsection{Software development in BBB location tracking system and GPS fencing}

Linux software has been used to develop in BBB location tracking system and GPS fencing. To calculate the distance between the two coordinates, equirectangular approximation has been used. The formula below shows how to calculate the distance between two points:

Formula

defined:

$$
\begin{gathered}
\mathrm{x}=\Delta \lambda \cdot \cos \varphi \mathrm{m} \\
\mathrm{y}=\Delta \varphi \\
\mathrm{d}=\mathrm{R} \cdot \sqrt{ }\left(\mathrm{x}^{2}+\mathrm{y}^{2}\right)
\end{gathered}
$$

$$
\begin{aligned}
& \operatorname{var} \mathrm{x}=(\lambda 2-\lambda 1) * \text { Math.cos }((\varphi 1+\varphi 2) / 2) \\
& \operatorname{var} \mathrm{y}=(\varphi 2-\varphi 1) \\
& \operatorname{var} \mathrm{d}=\text { Math.sqrt }\left(\mathrm{x}^{*} \mathrm{x}+\mathrm{y} * \mathrm{y}\right) * \mathrm{R} \\
& \operatorname{var} \mathrm{R}=6371 \mathrm{e} 3 \text { in metres } \\
& \operatorname{var} \varphi 1=\text { lat1 in Radians } \\
& \operatorname{var} \varphi 2=\text { lat2 in Radians } \\
& \operatorname{var} \Delta \varphi=(\text { lat2-lat1) in Radians } \\
& \operatorname{var} \Delta \lambda=(\text { lon2-lon1) in Radians }
\end{aligned}
$$

\subsubsection{Development of ThingSpeak}

ThingSpeak is an open source "Internet of Things" application and API to store and retrieve data from things using HTTP over the Internet or via a Local Area Network. With ThingSpeak, the user can create sensor 
logging applications, location tracking applications, and a social network of things with status updates. Data from the Beagle-Bone Black that contain GPS location will be transmit into ThingSpeak. This ThingSpeak will display into graph that can be easier to analyse.In the ThingSpeak, react name has been used as react PushingBox. PushingBox is a cloud that can send notifications based on API calls. From one request, it can send several notifications like a Push, a Tweet, an Email, message and others. This react can be notification to the parents by using message. From the Figure 13, the initial or origin of coordinate can be put at the latitude and longitude part. Apart from that, it also can set how further we need to notify from the origin coordinate.

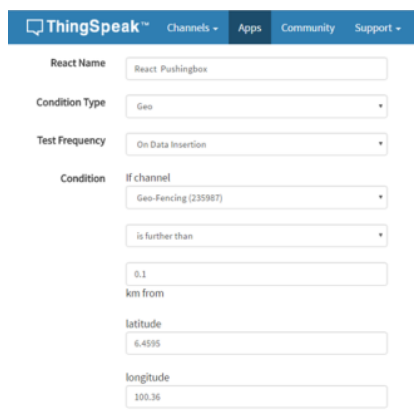

Fig.13. Development for the apps part.

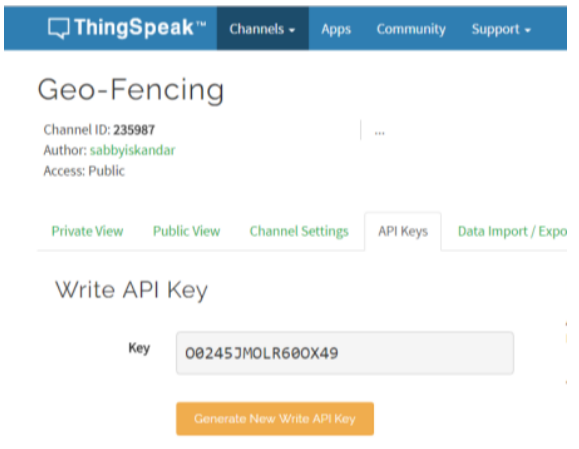

Fig.14. API key.

Figure 14 shows the API key for the ThingSpeak. It also displayed the channel ID. This API key is very important because it will be put on the URL that needed from the apps development by using MIT apps inventor.

\subsubsection{Application development in smartphone}

This MIT App inventor will divide into two parts, first part for the design layout on the screen and the second part for the block layout.

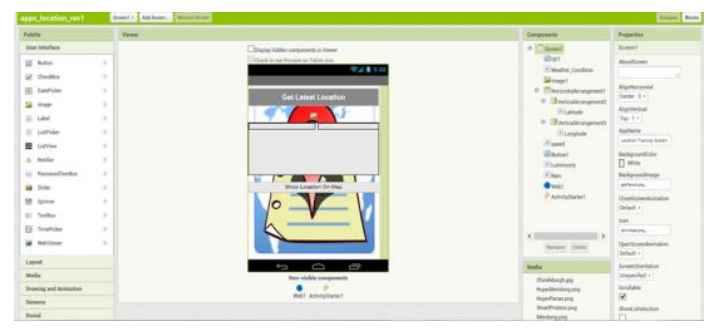

Fig.15. Design layout.

Figure 15 show the design layout for the apps. The design layouts contain the user interface, layout, media, drawing and animation, sensors and social. Each palette can be design depend on the designer desire by referring their own project. Anything can be change on the properties and component part.

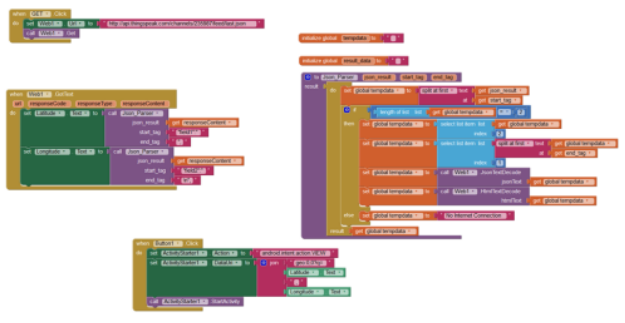

Fig.16. Block layout.

The Figure 16 shows the block layout for the apps design. The block displays the button in the the block form with the different colour. The URL from the web at ThingSpeak will be inside the block. The function for the URL is to call the ThingSpeak web and get the data from that web.

\subsubsection{Application development to give notification to parents}

Apart from the apps, parents will get the notification by the message with the voice message by using the Newtifry apps. Parents or user need to install the Newtifry from the play store into their smartphone. Newtifry is a push notification service to send server notifications to Android Mobile phones, running android. It uses Google Cloud Messaging APIs. It is intended to replace notifications sent by SMS. SMS cost money to send, and for single user applications this can give you real time notifications for free. Unlike other systems that do the server monitoring for user, Newtifry is just intended to get the message and voice message to the phone and no more. Newtifry is meant to be very simple and just focus on getting the notifications to the phone. User can plug Newtifry into your existing monitoring system to get the notifications going. Users supply the system administrators with a single unique key that identifies at message source, which they can enable or disable at will. 


\section{Result and Discussion}

In this part, the result can be review from the BBB, ThingSpeak, Apps and message notification. The coordinate from the reading of GPS antenna will be sent to the ThingSpeak in a graph form. From the graph at the ThingSpeak, the data can be an analyse it. After that, the data will be sent to the apps in the smartphone and sent the message notification.

\subsection{Result monitored in $B B B$}

Result from the BBB will display on the terminal by using Linux software. The terminal will show the value of latitude and longitude at the current position. The Figure 17 shows the terminal from the Linux software.

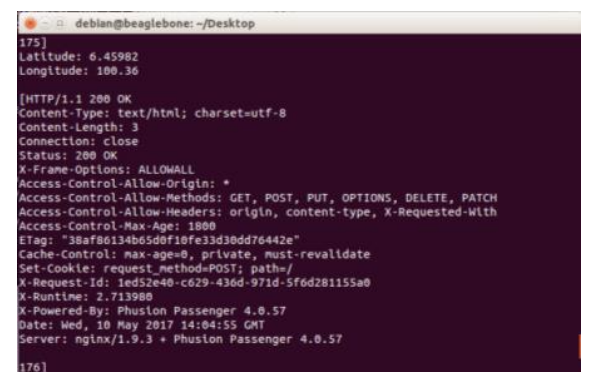

Fig.17. Terminal from the Linux software.

\subsection{Result monitored in ThingSpeak}

In this ThingSpeak, it will divide the chart into two parts. It contains GPS latitude and GPS longitude. The Figure 18 shows the GPS Latitude set for the origin while the Figure 19 shows the GPS Latitude for latest coordinate. From the graph below, $\mathrm{x}$-axis will display date while the $y$-axis will display value of latitude. It can see from the graph, each dot with red colour represent the movement of the children. From the graph, it can be easily to see and analyse.

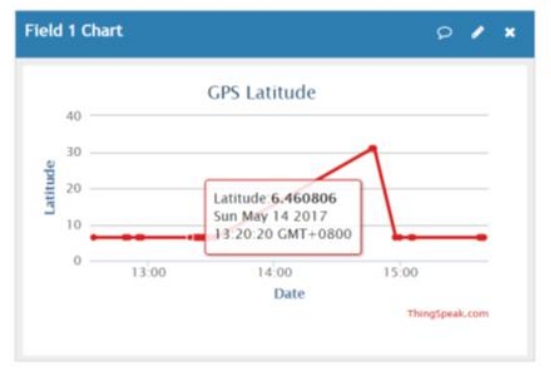

Fig.18. GPS Latitude for the origin.

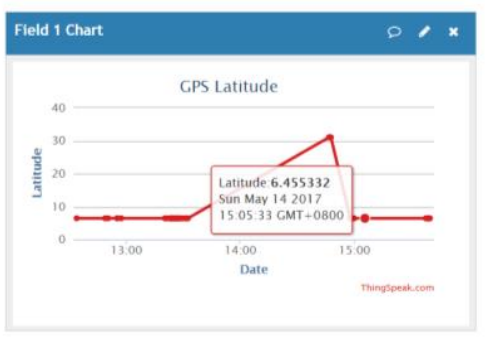

Fig.19. GPS Latitude for the latest coordinate.

The Figure 20 shows the GPS Longitude for the origin while Figure 21 shows the GPS Longitude for the latest coordinate. From the graph below, $\mathrm{x}$-axis will display the date while the $y$-axis will display value of Longitude. From the graph below, $\mathrm{x}$-axis will display date while the $y$-axis will display value of Longitude. It can see from the graph, each dot with red colour represent the movement of the children. From the graph, it can be easily to see and analyse.

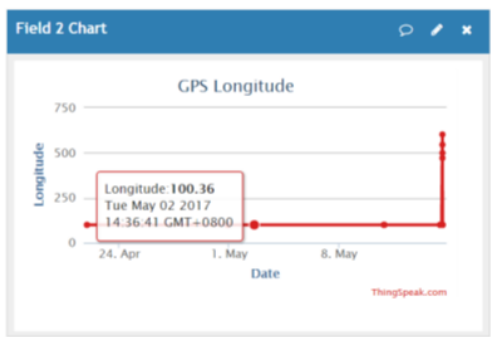

Fig.20. GPS Longitude for the origin.

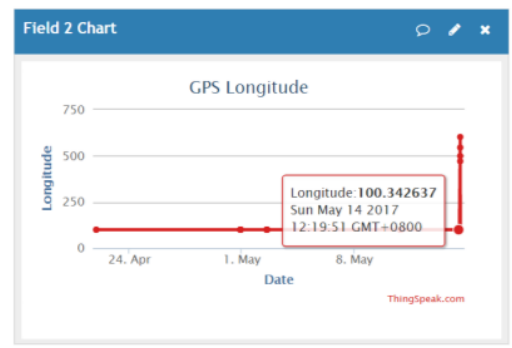

Fig.21. GPS Longitude for the latest coordinate.

The coordinate from the origin to the latest coordinate as shown as the graph above is more than 100 meters. Then, notification message will be sent to the parents and the latest coordinate also will display in the apps.

\subsection{Result monitored on Apps}

The apps is named as Location tracking system for children. This apps display the GPS latitude and longitude. For the additional part, we show the location on map based on the latest GPS coordinate. This location on map will help the parents share the location to the police, ambulance or any rescue bodies if any accidents happen to their children. The Figure 22 shows the icon apps in the smartphone. This application same with the other apps that already existed in the regular smartphone. 


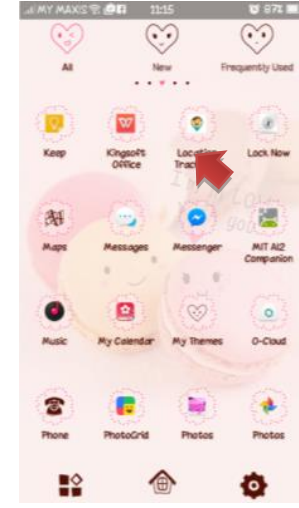

Fig.22. Icon in the smartphone.

The Figure 23 shows the design layout for the apps. After click the icon part, the apps will display as the Figure 23. When the user clicks the button "Get Latest Location", the coordinate will display in the form as latitude and longitude. From the design layout, it will show the latitude value is 6.4544 and the longitude value is 100.36. That coordinate shows the latest location for the children. There is a button "Show Location on Map" at the middle part. After the user knows the latest coordinate for their children, they can click that button "Show Location on Map". This button will display directly to the address in a goggle map exactly like in the Figure 23 by referring the current coordinate.

This location in Google map will help the parents send the location immediately to the police, ambulance or others rescue bodies if anything happens for their children. Figure 24 shows the Location in Google map.

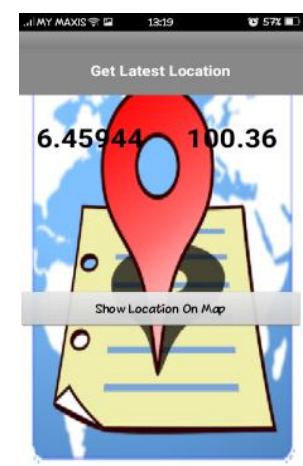

Fig.23. Design apps layout.

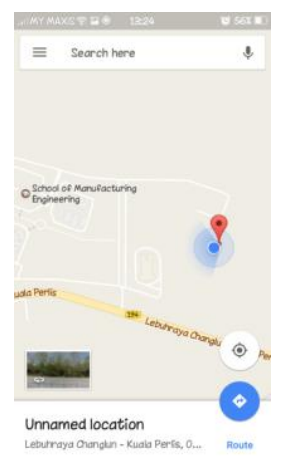

Fig.24. Location in Google map.

\subsection{Result monitored on notification message}

Apart from the apps, the user or parents will receive the message notification from the Newtifry. The notification will display the date and time as show at the Figure 25. The voice message will produce simultaneously with the message notification. After a click, the source as shown in the Figure 26, the detail message will display. The message will display "Your children is away from your home" as shown at the Figure 26.

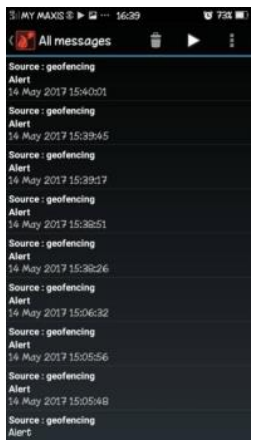

Fig.25. Message notification from Newtifry.

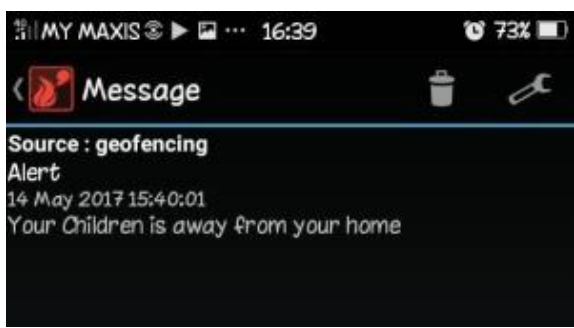

Fig.26. Message details.

\section{Conclusion and Future Work}

A prototype of a location and information tracking system for children is developed and presented. The GPS antenna is validated to be capable of acquiring coordinate data for the children and them wirelessly to the microcomputer. The microcomputer is able to accept these data and channel them to android apps through the smartphone. Parents as the user of location and information tracking system is able to monitor the movement of their children based on android apps.

\section{References}

1. A. B. Mieee, "GPS , GPRS, GIS FOR Tracking System," vol. 1, no. 8, pp. 527-529, 2011.

2. S. Holtmanns, S. P. Rao, and I. Oliver, "User location tracking attacks for LTE networks using the interworking functionality," 2016 IFIP Netw. Conf. (IFIP Networking) Work. IFIP Netw. 2016, pp. 315-322, 2016. 
3. A. Nayyar and V. Puri, "A Comprehensive Review of BeagleBone Technology: Smart Board Powered by ARM," vol. 10, no. 4, pp. 95-108, 2016.

4. "Construction Research Congress 2012 (C) ASCE 2012 818," pp. 818-827, 2012.

5. G. Cardone, A. Cirri, A. Corradi, L. Foschini, R. Ianniello, and R. Montanari, "Crowdsensing in Urban areas for city-scale mass gathering management: Geofencing and activity recognition," IEEE Sens. J., vol. 14, no. 12, pp. 4185-4195, 2014.

6. A. K. Kamaraj Radha M Priyanka M Punitha Asst, "Intelligent Transport System Using Integrated GPS Optimized Reader," pp. 332336, 2016. 